\title{
The broad-band X-ray spectrum of the dipping low mass X-ray binary EXO 0748-676
}

\author{
L. Sidoli ${ }^{1}$, A. N. Parmar ${ }^{2}$, and T. Oosterbroek ${ }^{3}$ \\ ${ }^{1}$ Istituto di Astrofisica Spaziale e Fisica Cosmica - Sezione di Milano "G. Occhialini” - IASF/CNR, via Bassini 15, \\ 20133 Milano, Italy \\ e-mail: sidoli@mi.iasf.cnr.it \\ 2 Astrophysics Mission Division, Research and Scientific Support Department of ESA, ESTEC, Postbus 299, \\ 2200 AG Noordwijk, The Netherlands \\ 3 INTEGRAL Science Operations Centre, Science Operations and Data Systems Division, Research and Scientific Support \\ Department of ESA, ESTEC, Postbus 299, 2200 AG Noordwijk, The Netherlands
}

Received 7 January 2004 / Accepted 13 August 2004

\begin{abstract}
We present results of a $0.1-100 \mathrm{keV}$ BeppoSAX observation of the dipping low-mass X-ray binary (LMXRB) EXO 0748-676 performed in 2000 November. During the observation EXO 0748-676 exhibited X-ray eclipses, type I X-ray bursts and dipping activity over a wide range of orbital phases. The $0.1-100 \mathrm{keV}$ "dip-free"(i.e. dipping and eclipsing intervals excluded) spectrum is complex, especially at low energies where a soft excess is present. Two very different spectral models give satisfactory fits. The first is the progressive covering model, consisting of separately absorbed black body and cut-off power law components. This model is often used to study the dipping activity in LMXRB. The second model is an absorbed cut-off power law together with a moderately ionized absorber with a sub-solar abundance of Fe and a $2.13 \mathrm{keV}$ absorption feature (tentatively identified with Si XIII). This ionized absorber may be the same feature as seen by Chandra during dips from EXO 0748-676. The fact that these two physically very different models both give acceptable fits to the data and plausible values of the best fit parameters indicates how difficult it is to reliably model such complex dipping spectra with moderate spectral resolution data. The blackbody component required by the progressive covering LMXRB model probably has a more complex underlying nature, due to the strong effects of ionized absorption present.
\end{abstract}

Key words. accretion, accretion disks - stars: individual: EXO 0748-676 - X-rays: general

\section{Introduction}

EXO 0748-676 is a transient low-mass X-ray binary (LMXRB) discovered with EXOSAT (Parmar et al. 1986). It shows $\mathrm{X}$-ray eclipses every $3.82 \mathrm{hr}$, complex dipping activity and type I X-ray bursts, from which a distance of $10 \mathrm{kpc}$ has been estimated (Parmar et al. 1986; Gottwald et al. 1986). Quasi-periodic oscillations (QPOs) at $\mathrm{Hz}$ and $\mathrm{kHz}$ frequencies were discovered using R-XTE (Homan et al. 1999; Homan \& van der Klis 2000). A summed XMM-Newton Reflection Grating Spectrometer (RGS) spectrum of 28 type I bursts from EXO 0748-676 revealed absorption features identified with Fe XXV and XXVI and O VIII transitions all with a gravitational redshift of 0.35 (Cottam et al. 2002). For the astrophysically plausible range of neutron star masses the measured redshift is completely consistent with models of neutron stars composed of normal neutral matter, while it excludes some models in which neutron stars are made of more exotic matter.

The spectral and temporal complexity of the soft Xray spectrum of EXO0748-676 was already hinted at in early ROSAT and ASCA observations (Schulz 1999;
Thomas et al. 1997; Church et al. 1998). The vastly improved narrow line sensitivity of the RGS has allowed the $0.3-2.5 \mathrm{keV}$ X-ray spectrum of EXO 0748-676 to be studied in detail revealing the presence of both absorption and emission features from ionized $\mathrm{Ne}, \mathrm{O}$ and $\mathrm{N}$. The line producing material is probably located well away from the orbital plane (Cottam et al. 2001). The lines are broadened $\left(\sim 1000 \mathrm{~km} \mathrm{~s}^{-1}\right)$ and exhibit a direct correlation between the velocity broadening and the degree of ionization. The velocity shift of the emitting plasma is $<300 \mathrm{~km} \mathrm{~s}^{-1}$. The RGS lightcurve does not show any orbital modulation, indicating that the source of the soft continuum is extended. $0.3-10 \mathrm{keV}$ observations obtained with the XMM-Newton European Photon Imaging Camera (EPIC) revealed a varying lightcurve with soft X-ray flares together with dipping activity, X-ray eclipses and type I X-ray bursts (Bonnet-Bidaud et al. 2001). The "persistent" (i.e. dipping and eclipsing intervals excluded) spectrum was interpreted using a two-component model consisting of an extended thermal $(k T=0.64 \mathrm{keV})$ component with a radius of $3 \times 10^{10} \mathrm{~cm}$ and a power law with a photon index, $\Gamma$, of 1.35 . The power law is affected by additional absorption, $N_{\mathrm{H}}$, of $6.4 \times 10^{22} \mathrm{~cm}^{-2}$ and is 
probably produced in a more compact Comptonizing accretion disk corona with a radius of $2 \times 10^{8} \mathrm{~cm}$. The thermal component may have strongly non-solar abundances of $\mathrm{N}(10 \pm 2)$, $\mathrm{O}(0.6 \pm 0.1), \mathrm{Ne}(0.02 \pm 0.02)$ and $\mathrm{Mg}(0.31 \pm 0.23)$.

A Chandra observation of EXO 0748-676 with the HighEnergy Transmission Grating Spectrometer (HETGS) revealed several strong discrete spectral signatures of a photo-ionized plasma in orbit around the neutron star (Jimenez-Garate et al. 2003). Recombination lines from $\mathrm{H}$-like and He-like $\mathrm{O}, \mathrm{Ne}$ and $\mathrm{Mg}$ were detected, with a mean velocity broadening of $\sim 750 \mathrm{~km} \mathrm{~s}^{-1}$. The continuum requires compact soft and hard sources together with an extended recombination region associated with the thickened outer regions of the accretion disk where the accretion stream impacts. The spectral changes during dips require the presence of both a neutral and an ionized absorber (Jimenez-Garate et al. 2003).

\section{Observations and data reduction}

EXO 0748-676 was observed with BeppoSAX between 2000 November 4, 16:52 and November 6, 07:44 UTC, with an onsource time of $66 \mathrm{ks}$. We present results from the Low-Energy Concentrator Spectrometer (LECS; 0.1-10 keV; Parmar et al. 1997), the Medium-Energy Concentrator Spectrometer (MECS; 1.8-10 keV; Boella et al. 1997), High Pressure Gas Scintillation Proportional Counter (HPGSPC; 5-120 keV; Manzo et al. 1997) and the Phoswich Detection System (PDS; 15-300 keV; Frontera et al. 1997) instruments. All these instruments are coaligned and collectively referred to as the Narrow Field Instruments. The MECS and the LECS are grazing incidence telescopes with imaging gas scintillation proportional counters in their focal planes. The non-imaging HPGSPC consists of a single unit with a collimator that was alternatively rocked on- and $180^{\prime}$ off-source every $96 \mathrm{~s}$ during most of the observations. During the observations in the year 2000 the collimator remained on-source. The PDS consists of four independent non-imaging units arranged in pairs each having a separate collimator. Each collimator was alternatively rocked on- and $210^{\prime}$ off-source every $96 \mathrm{~s}$ during the observations. The data were reprocessed using the SAX Data Analysis System (SAXDAS) version 2.0.0. For the spectral analysis counts have been extracted from circular regions with standard radii ( $4^{\prime}$ for the MECS and $8^{\prime}$ for the LECS). The LECS and MECS spectra were rebinned to oversample the full width half maximum of the energy resolution by a factor 3 and to have additionally a minimum of 20 counts per bin to allow use of $\chi^{2}$ statistics. The HPGSPC and PDS spectra were rebinned using the standard techniques in SAXDAS. Response matrices appropriate for the sizes of the extraction regions were used.

\section{Results}

EXO 0748-676 displays many kinds of variability such as X-ray eclipses, dips and type I X-ray bursts (Fig. 1). In order to study the EXO0748-676 spectrum without the effects of this variability it is necessary to identify dip-free intervals (the bursts and eclipses are easy to detect). Homan et al. (2003)

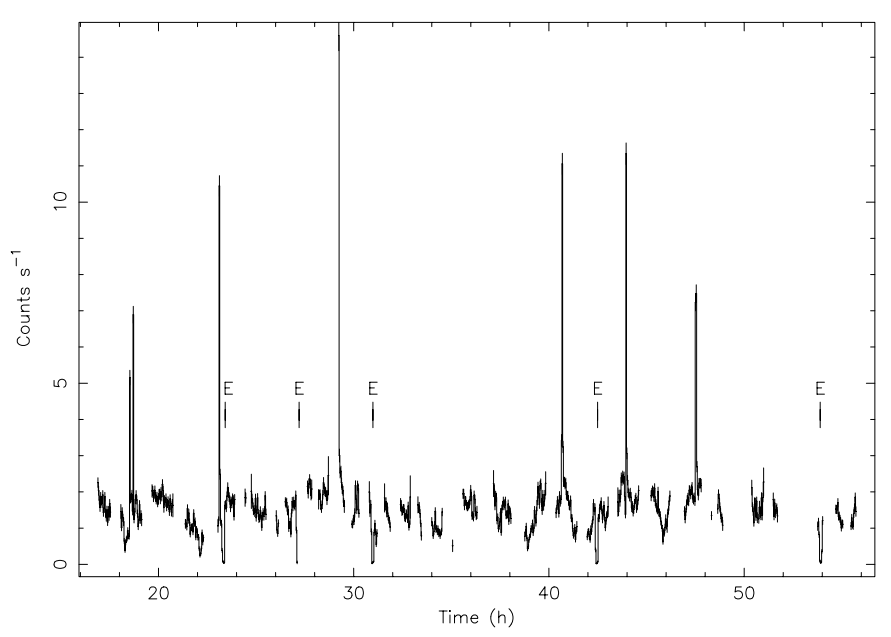

Fig. 1. EXO 0748-676 MECS 1.8-10 keV lightcurve with a binning of $128 \mathrm{~s}$. A double burst and 5 single bursts are clearly visible together with eclipses every $3.8 \mathrm{~h}$ (marked by the letter "E").

show that EXO 0748-676 can display low-level dipping activity which is not easily visible above $2 \mathrm{keV}$, but which can affect the overall spectrum. With this result in mind, lightcurves in two different energy ranges were produced (Fig. 2) with the bursting intervals excluded. Examination of the $0.1-2 \mathrm{keV}$ LECS lightcurve (Fig. 2, upper panel) reveals strong variability throughout the observation. This emphasizes the difficulty in identifying dip-free intervals using only a visual inspection of the source lightcurves. In order to investigate whether variations in the source hardness ratio provide a reliable means of identifying dip-free intervals, the LECS $2-10 \mathrm{keV}$ counts were divided by those between $0.1-2 \mathrm{keV}$ to create hardness ratios which were plotted against the $0.1-2.0 \mathrm{keV}$ count rate (Fig. 3). The dipping intervals stand out clearly owing to their higher hardness ratios. For count rates $\gtrsim 0.2 \mathrm{~s}^{-1}$ the hardness ratio does not vary appreciably. Thus, by selecting events when the count rate was above this intensity threshold, it is possible to obtain the dip-free EXO 0748-676 spectrum. Additional confidence that this selection corresponds to the "dip-free" spectrum is obtained from considering spectra extracted from intervals corresponding to nearby intensity selections (e.g., $0.2-0.3$ count s$^{-1}$, $0.3-0.4$ count $\mathrm{s}^{-1}$, and $>0.4$ count $\mathrm{s}^{-1}$ ). These spectra do not show significant differences from that extracted from intervals with $>0.2$ count $\mathrm{s}^{-1}$.

Thus, the extraction of an eclipse and dip free spectrum was performed using only time intervals where the source displays a constantly low hardness ratio, corresponding to intervals when the LECS $0.1-2 \mathrm{keV}$ count rate was $>0.2 \mathrm{~s}^{-1}$ when rebinned on a timescale of $128 \mathrm{~s}$. Since the LECS was only operated during spacecraft night when the other 3 instruments were also operated, the same time intervals were then used to extract corresponding MECS, HPGSPC and PDS spectra, leading to exposure times of $4.3 \mathrm{ks}$ for all 4 instruments.

The data were then selected in the energy ranges 0.1-8.0 keV (LECS), 1.8-10 keV (MECS), 8-20 keV (HPGSPC) and 15-100 keV (PDS) and rebinned using standard procedures. The resulting background-subtracted count rates were $0.9,1.6,2.3$ and $3.4 \mathrm{~s}^{-1}$ for the LECS, MECS, 


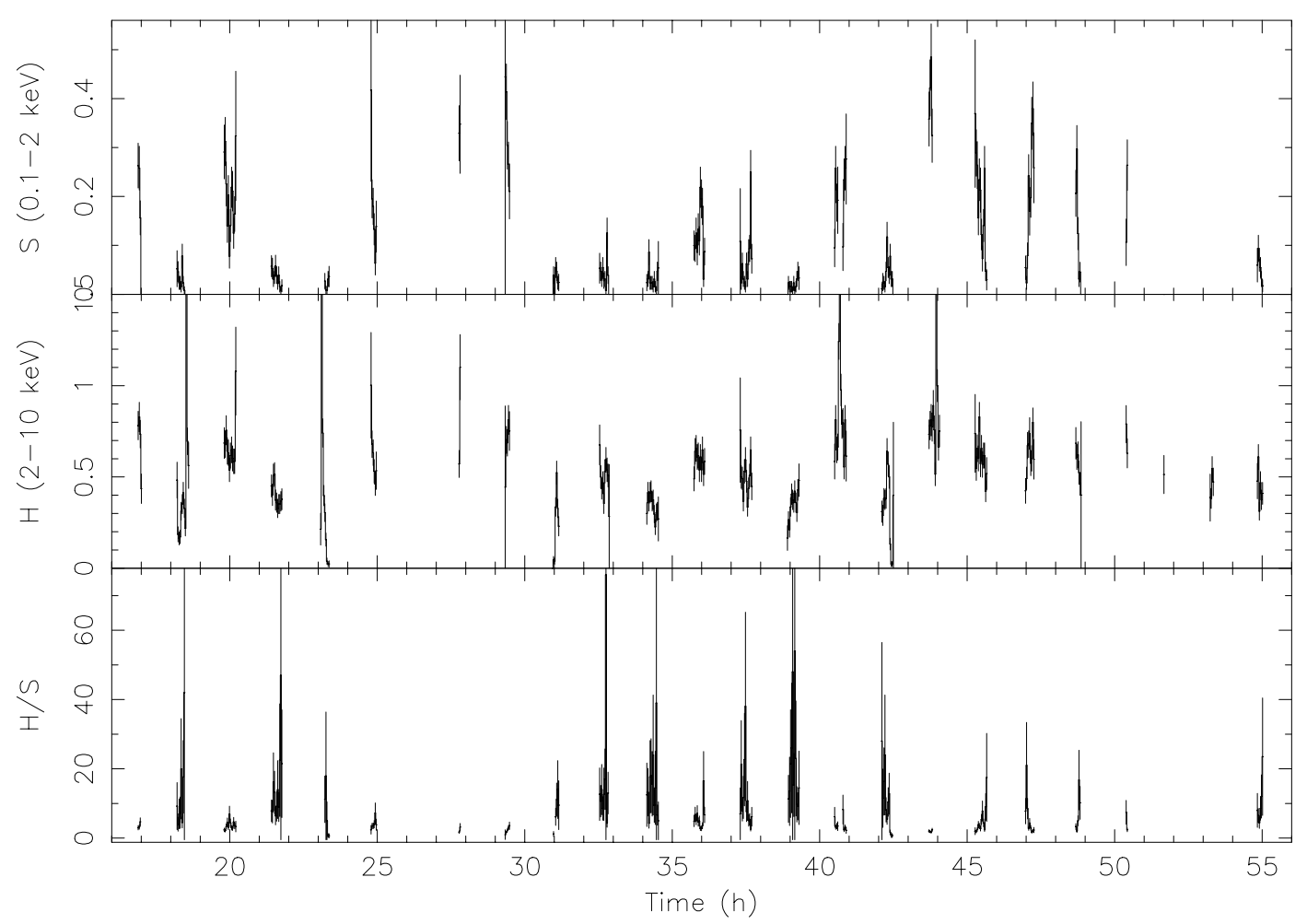

Fig. 2. EXO 0748-676 LECS 0.1-2 keV (“S”, upper panel) and LECS 2-10 keV ("H”, middle panel) lightcurves, with bursting intervals excluded. The lower panel shows the hardness ratio, H/S. The binning time is $128 \mathrm{~s}$.

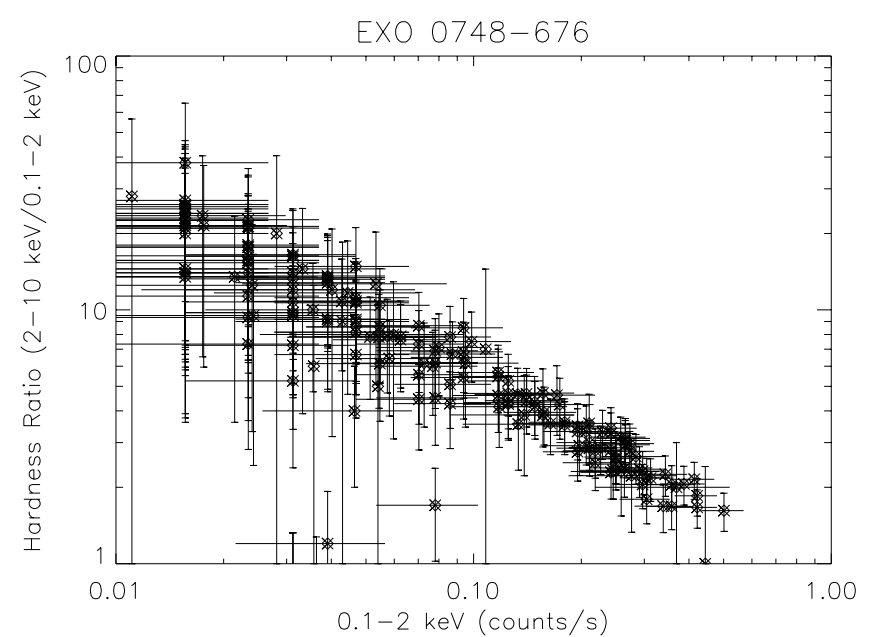

Fig. 3. Hardness ratio (2-10 keV LECS counts divided by those between $0.1-2 \mathrm{keV}$ ) versus the LECS $0.1-2 \mathrm{keV}$ intensity. Above a $0.1-2 \mathrm{keV}$ count rate of around $0.2 \mathrm{~s}^{-1}$ the hardness ratio does not vary appreciably with count rate.

HPGSPC and PDS, respectively. In the spectral fitting, factors were included to account for normalization uncertainties between the instruments. These factors were constrained to be within their usual ranges during the fitting. All spectral uncertainties and upper-limits are given at $90 \%$ confidence. Spectral analysis was performed with the XSPEC v.11 software package.

EXO0748-676 is detected in the PDS up to an energy of $100 \mathrm{keV}$. An absorbed power law model does not account for the broad-band emission, leaving large positive residuals below $1 \mathrm{keV}$ and around $20 \mathrm{keV}$, together with negative residuals $\gtrsim 30 \mathrm{keV}$, indicative of a high-energy cut-off $\left(\chi^{2}=892\right.$ for 176 degrees of freedom, d.o.f.). Adopting a cut-off power law model resulted in a significantly better fit $\left(\chi^{2} /\right.$ d.o.f. $\left.=407 / 175\right)$, although the fit is still unacceptable because of the presence of a large soft excess below $1 \mathrm{keV}$. We note that a satisfactory fit $\left(\chi^{2} /\right.$ d.o.f. $\left.=160.1 / 154\right)$ to the $2-100 \mathrm{keV}$ spectrum can be obtained with an absorbed cut-off power law model with $N_{\mathrm{H}}=2 \times 10^{22} \mathrm{~cm}^{-2}, \Gamma=1.3$ and a cut-off energy, $E_{\mathrm{c}}$, of $44 \mathrm{keV}$.

The 0.1-100 keV spectrum was then modeled using different types of two-component models, always including the cut-off power law to account for the high energy part of the spectrum. The soft excess was modeled using black-body and multicolor disk-blackbody (Mitsuda et al. 1984) components, an ionized absorber and partial covering absorption. However, unacceptable fits $\left(\chi^{2}\right.$ red $\left.>1.4\right)$ were always obtained. The XMM-Newton observations reported in Bonnet-Bidaud et al. (2001) revealed a complex persistent spectrum, deconvolved with a two-component model consisting of a RaymondSmith (RS) thermal emission $\left(k T_{\mathrm{RS}}=0.64 \mathrm{keV}\right)$ and a power law component $(\Gamma=1.35)$. The two components were differently absorbed with $N_{\mathrm{H}}=6.4 \times 10^{22} \mathrm{~cm}^{-2}$ for the power law, and $N_{\mathrm{H}}=0.15 \times 10^{22} \mathrm{~cm}^{-2}$ for the thermal plasma. This model was fit to our data, with the same parameters (but with the normalizations free), but it does not account well for the BeppoSAX data. A VMEKAL model (e.g., a thermal plasma model with variable metal abundances) was tried next, since Bonnet-Bidaud et al. (2001) require non-solar abundances of $\mathrm{N}(10 \pm 2), \mathrm{O}(0.6 \pm 0.1), \mathrm{Ne}(0.02 \pm 0.02)$ and $\mathrm{Mg}(0.31 \pm 0.23)$ in their fits. The abundances of $\mathrm{N}, \mathrm{O}, \mathrm{Ne}$ and $\mathrm{Mg}$ were allowed 
to vary during the fitting, but could not be usefully constrained, nor a significantly better fit obtained. Large positive residuals below $1 \mathrm{keV}$ still are present.

Another model successfully used in the modeling of EXO 0748-676 spectra (and other dipping LMXRBs) was introduced by Church et al. (1998) and consists of a blackbody and an extended Comptonizing corona described by a power law spectrum (progressively covered by absorbing matter during dipping activity). Applying this model to the $0.1-100 \mathrm{keV}$ "dip-free" emission resulted in a good description of the spectrum $\left(\chi^{2} /\right.$ d.o.f. $\left.=185.7 / 170\right)$. Contrary to the "standard" Church et al. (1998) model, where the "dip-free" emission is un-covered, extra absorption of the Comptonized component (see Table 1 and Fig. 4) is required to obtain a satisfactory fit to the data.

Finally, we tested the possibility that the soft excess is due to the presence of an ionized absorber. Adopting the ABSORI model in XSPEC (Done et al. 1992) and fixing the ionizing power law photon index to that of the cut-off power law component, we obtained a satisfactory fit $\left(\chi^{2} /\right.$ d.o.f. $\left.=203.2 / 173\right)$ to the data with the following parameters: interstellar column density $N_{\mathrm{H}}=10^{21} \mathrm{~cm}^{-2}$, an intrinsic absorber with a column density $N_{\text {absori }}=(5 \pm 1) \times 10^{22} \mathrm{~cm}^{-2}$, an absorber ionization state $\xi=350 \pm 90 \mathrm{erg} \mathrm{cm} \mathrm{s}^{-1}\left(\xi=L / n R^{2}\right)$, a cut-off power law with $\Gamma=1.3 \pm 0.1$ and $E_{\mathrm{c}}=45 \pm 10 \mathrm{keV}$. Since there are still strong negative residuals around $2.1-2.2 \mathrm{keV}$, we included a narrow negative Gaussian in the model. This led to a significantly better fit $\left(\chi^{2} /\right.$ d.o.f. $\left.=184.4 / 170\right)$, with ABSORI and cut-off power law parameters substantially unchanged, plus an absorption feature with a centroid at $2.13 \mathrm{keV}$ and an equivalent width, $E W$, of $\sim 50 \mathrm{eV}$, which can be tentatively identified with absorption from highly ionized $\mathrm{Si}$. We note however that this energy is close to the Au l-edges of the BeppoSAX mirrors and we cannot exclude that this feature is due to incorrect instrumental modeling. A better fit can be found if the Fe abundance in the ionized absorber is allowed to vary, obtaining a sub-solar abundance of Fe (see Table 2 and Fig. 5).

\section{Discussion}

We have performed for the first time broad-band spectroscopy of the dipping, eclipsing, LMXRB EXO 0748-676. The X-ray spectrum extends to at least $100 \mathrm{keV}$ and exhibits a cut-off at $50 \pm 10 \mathrm{keV}$. At low energies, the spectrum is particularly complex with a soft excess that cannot be easily fit. Various combinations of simple models were tried without success. However, we did find that two models can adequately fit the data. In both cases the high-energy component is modeled by a cut-off power law, and the difference is primarily in how the low-energy part of the spectrum is modeled. In the first case, following Church et al. (1998) this is as an absorbed blackbody and additional partial covering of the cut-off power law is required. Contrary to the Church et al. (1998) model for dipping LMXRB, additional absorption on the cut-off power law component is required. In the second model, an additional additive component is not required and instead the cut-off power law is absorbed by moderately ionized material with a sub-solar Fe abundance. A $2.13 \mathrm{keV}$ absorption feature, which may be
Table 1. Parameters for the $0.1-100 \mathrm{keV}$ BeppoSAX "dip-free" emission spectrum of EXO 0748-676 fitted with the Church et al. (1998) model. $N_{\mathrm{H}}$ is the interstellar column density, $N_{\mathrm{H}}(\mathrm{BB})$ and $N_{\mathrm{H}}(\mathrm{POW})$ are the column densities of the blackbody and power law components, $f$ is the covering fraction, $\Gamma$ is the cut-off power law photon index, and $E_{\mathrm{c}}$ is the high energy cut-off. Fluxes are uncorrected for absorption. The luminosity is only corrected for interstellar absorption and is for a distance of $10 \mathrm{kpc}$.

\begin{tabular}{|c|c|}
\hline Parameter & Value \\
\hline$N_{\mathrm{H}}(\mathrm{BB})\left(10^{22} \mathrm{~cm}^{-2}\right)$ & $0.12 \pm 0.01$ \\
\hline$k T_{\mathrm{bb}}(\mathrm{keV})$ & $0.13_{-0.01}^{+0.02}$ \\
\hline$R_{\mathrm{bb}}(\mathrm{km})$ & $<100^{-0.01}$ \\
\hline$N_{\mathrm{H}}\left(10^{22} \mathrm{~cm}^{-2}\right)$ & $0.6 \pm 0.3$ \\
\hline$N_{\mathrm{H}}(\mathrm{POW})\left(10^{22} \mathrm{~cm}^{-2}\right)$ & $5.4_{-1.7}^{+1.9}$ \\
\hline$f$ & $0.4_{-0.1}^{+0.2}$ \\
\hline$\Gamma$ & $1.34_{-0.07}^{+0.12}$ \\
\hline$E_{\mathrm{c}}(\mathrm{keV})$ & $50 \pm 10$ \\
\hline Flux $(2-10 \mathrm{keV})\left(\mathrm{erg} \mathrm{cm}^{-2} \mathrm{~s}^{-1}\right)$ & $1.7 \times 10^{-10}$ \\
\hline Flux $(0.1-100 \mathrm{keV})\left(\mathrm{erg} \mathrm{cm}^{-2} \mathrm{~s}^{-1}\right)$ & $6.8 \times 10^{-10}$ \\
\hline Luminosity $(2-10 \mathrm{keV})\left(\mathrm{erg} \mathrm{s}^{-1}\right)$ & $1.7 \times 10^{36}$ \\
\hline Luminosity $(0.1-100 \mathrm{keV})\left(\mathrm{erg} \mathrm{s}^{-1}\right)$ & $7.7 \times 10^{36}$ \\
\hline$\chi^{2} /$ d.o.f. & $185.7 / 170$ \\
\hline
\end{tabular}

Table 2. Best fit parameters for the 0.1-100 keV BeppoSAX “dipfree" spectrum of EXO 0748-676 fitted with a cut-off power law, absorbed by an ionized absorber with a non-solar abundance of Fe. $N_{\mathrm{H}}$ is the interstellar column density, $N_{\text {absori }}$ the local absorbing column density due to the ionized absorber where $\xi$ is the ionization parameter $\left(\xi=L / n R^{2}\right.$, where $L$ is the luminosity of the illuminating source, $n$ is the absorbing plasma density and $R$ is the distance of the absorbing material from the ionizing source), $\Gamma$ is the cut-off power law photon index and $E_{\mathrm{c}}$ is the high energy cut-off. $E_{\text {line }}, \sigma$ and $I_{\text {line }}$ are the absorption line energy, width and intensity, respectively. Fluxes are uncorrected for absorption. The luminosity has been corrected only for interstellar absorption and is for a distance of $10 \mathrm{kpc}$.

\begin{tabular}{|c|c|}
\hline Parameter & Value \\
\hline$N_{\mathrm{H}}\left(10^{22} \mathrm{~cm}^{-2}\right)$ & $0.11_{-0.04}^{+0.04}$ \\
\hline$N_{\text {absori }}\left(10^{22} \mathrm{~cm}^{-2}\right)$ & $8_{-3}^{+5.04}$ \\
\hline$\xi$ & $450_{-140}^{+170}$ \\
\hline Fe abundance & $0.23_{-0.21}^{+0.30}$ \\
\hline$\Gamma$ & $1.35_{-0.10}^{+0.10}$ \\
\hline$E_{\mathrm{c}}(\mathrm{keV})$ & $50 \pm 10$ \\
\hline$E_{\text {line }}(\mathrm{keV})$ & $2.13 \pm 0.06$ \\
\hline$\sigma(\mathrm{eV})$ & $<200$ \\
\hline$I_{\text {line }}\left(10^{-4}\right.$ photons $\left.\mathrm{cm}^{-2} \mathrm{~s}^{-1}\right)$ & $-6_{-6}^{+2}$ \\
\hline$E W(\mathrm{eV})$ & $-50_{-70}^{+10}$ \\
\hline Flux $(2-10 \mathrm{keV})\left(\mathrm{erg} \mathrm{cm}^{-2} \mathrm{~s}^{-1}\right)$ & $1.7 \times 10^{-10}$ \\
\hline Flux $(0.1-100 \mathrm{keV})\left(\mathrm{erg} \mathrm{cm}^{-2} \mathrm{~s}^{-1}\right)$ & $6.8 \times 10^{-10}$ \\
\hline Luminosity $(2-10 \mathrm{keV})\left(\mathrm{erg} \mathrm{s}^{-1}\right)$ & $1.7 \times 10^{36}$ \\
\hline Luminosity $(0.1-100 \mathrm{keV})\left(\mathrm{erg} \mathrm{s}^{-1}\right)$ & $7.2 \times 10^{36}$ \\
\hline$\chi^{2} /$ d.o.f. & $175.2 / 169$ \\
\hline
\end{tabular}

from highly ionized $\mathrm{Si}$ (Si XIII), or due to an inadequate modeling of the instrumental response, is also required. We note that the interstellar absorption determined with this model is consistent with the galactic value measured in this direction $\left(N_{\text {gal }}=1.1 \times 10^{21} \mathrm{~cm}^{-2}\right.$; Dickey \& Lockman 1990). The fact 

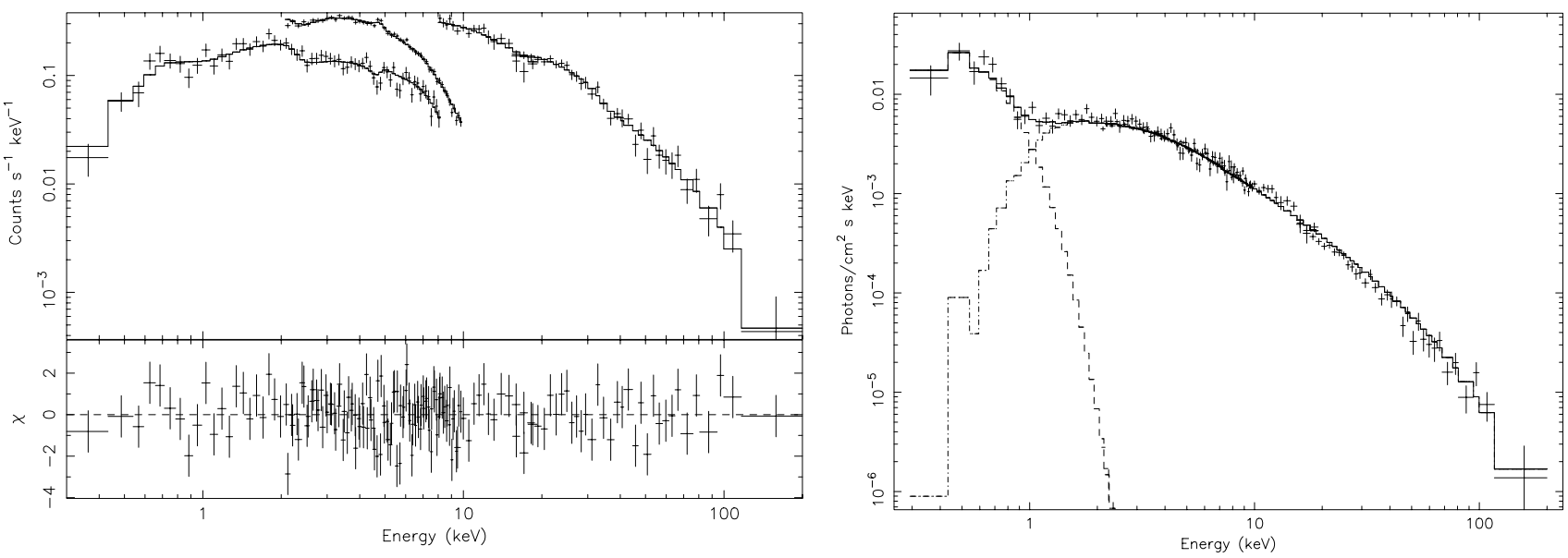

Fig. 4. 0.1-100 keV BeppoSAX spectrum of EXO 0748-676 fitted with the progressive covering model proposed by Church et al. (1998) (see Table 1 for the results). The left panels show the best fit count spectrum and the residuals (in units of standard deviation). The right panel shows the photon spectrum.
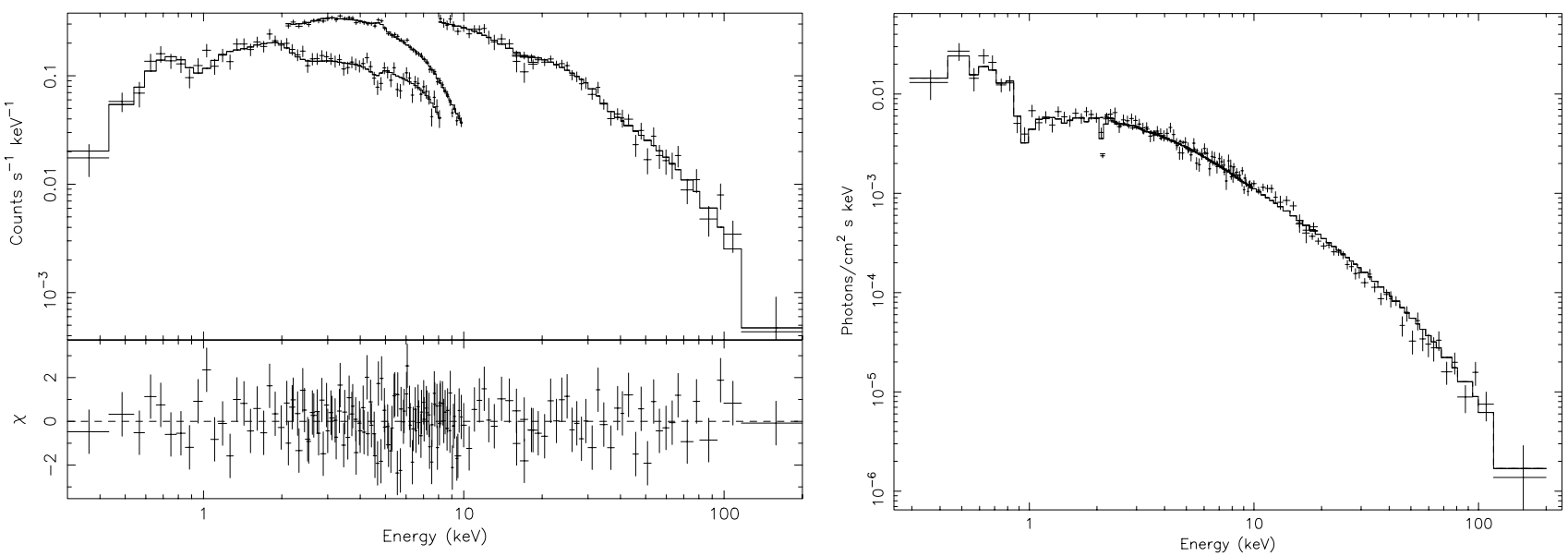

Fig. 5. 0.1-100 keV BeppoSAX spectrum of EXO0748-676 fitted with a cut-off power law and an ionized absorber (see Table 2 for the parameters). The left panels show the best fit count spectrum and the residuals (in units of standard deviation). The right panel shows the photon spectrum.

that these two physically very different models both give acceptable fits to the data and provide plausible values of the best fit parameters gives an indication of the complexity of the $\lesssim 2 \mathrm{keV}$ EXO 0748-676 spectrum and the difficulties of modeling this region using data obtained from moderate spectral resolution instruments. In addition, these result indicate that the blackbody component required by the Church et al. (1998) LMXRB model probably has a more complex underlying nature, due to the strong effects of ionized absorption present in this source.

The normalization of the high energy cut-off power law observed from EXO 0748-676 during the BeppoSAX observation is about 1.4 times higher than that during the XMM-Newton observation, performed about 7 months earlier (Bonnet-Bidaud et al. 2001). The slopes of the high-energy part of the spectrum in these two observations are compatible. The XMM-Newton observation demonstrated for the first time the existence of an extended soft thermal component from EXO 0748-676. We tried to fit the BeppoSAX observation with the XMM-Newton best fit, but were unsuccessful. Indeed, the
BeppoSAX 0.1-2 keV spectrum cannot be adequately fit with a hot thermal plasma model, even allowing a different absorption with respect to the high-energy part (as in the XMM-Newton observation). This probably means that during the BeppoSAX observation the extended thermal halo was suppressed, while the Componization component became 1.4 times brighter. The observed $0.5-10 \mathrm{keV}$ (uncorrected for absorption) flux during the BeppoSAX observation $\left(1.9 \times 10^{-10} \mathrm{erg} \mathrm{cm}^{-2} \mathrm{~s}^{-1}\right)$ is about twice that observed during the XMM-Newton observation $\left(1.1 \times 10^{-10} \mathrm{erg} \mathrm{cm}^{-2} \mathrm{~s}^{-1}\right)$.

If a thermal component similar to that observed in XMM-Newton spectrum were present in EXO 0748-676 during the BeppoSAX observation (with the same temperature of $0.6 \mathrm{keV}$ ), it should have had an additional absorption of $1.1 \times 10^{22} \mathrm{~cm}^{-2}$ or an emission measure, $A_{\mathrm{RS}},<3.3 \times 10^{-3}$ (where $A_{\mathrm{RS}}$ is in units of $10^{-14} /\left(4 \pi d^{2}\right) \int n_{\mathrm{e}} n_{\mathrm{H}} \mathrm{d} V$, where $d$ is the distance to the source $(\mathrm{cm}), n_{\mathrm{e}}$ is the electron density, and $n_{\mathrm{H}}$ is the hydrogen density). While the low abundance of $\mathrm{Fe}$ is consistent with the lack of any $\mathrm{Fe}$ line detection by Bonnet-Bidaud et al. (2001) with the XMM-Newton RGS, the 
presence of ionized $\mathrm{Si}$ is not, since no emission lines from $\mathrm{Si}$ were present in the RGS spectrum, leading the authors to conclude a very low abundance of this element. This difference could be due to a different ionization status of the absorbing matter, or as mentioned earlier, may be an instrumental artifact due to inadequate modeling of instrumental Au edges. The ionized absorber detected in the "dip-free" spectrum is probably the same as observed by Chandra (Jimenez-Garate et al. 2003) during dips, and interpreted by them as a thickened photoionized region located in the outer accretion disk. Our results indicate that the effects of this ionized absorber are also visible in the "dip-free" spectrum.

Using the best fit value of the ionization parameter of the absorbing matter, $\xi \sim 450 \mathrm{erg} \mathrm{cm} \mathrm{s}^{-1}\left(\xi=L / n R^{2}\right.$, where $L$ is the luminosity of the illuminating source, $n$ is the absorbing plasma density and $R$ is the distance of the absorbing matter from the ionizing source), and the $2-10 \mathrm{keV}$ luminosity $\left(2.0 \times 10^{36} \mathrm{erg} \mathrm{s}^{-1}\right)$, we obtain $n R^{2}=\sim 4 \times 10^{33} \mathrm{~cm}^{-1}$. Assuming that the absorbing plasma has the same dimensions as the corona $\left(2 \times 10^{8} \mathrm{~cm}\right.$ radius; Bonnet-Bidaud et al. 2001) we obtain a density of $\sim 10^{17} \mathrm{~cm}^{-3}$ for the absorbing ionized plasma. Alternatively, if we assume that this absorber is located in the outer accretion disk (as found with Chandra, Jimenez-Garate et al. 2003, $R \sim 10^{10} \mathrm{~cm}$ ), we find a density of $\sim 4 \times 10^{13} \mathrm{~cm}^{-3}$.

It is useful to compare our results with those of four XMM-Newton observations of EXO 0748-676 obtained in 2000-2001 recently reported in Homan et al. (2003). Due to the brightness of the source, serious pile-up problems occurred during the XMM-Newton observations both in the MOS and PN cameras, limiting the spectral analysis to only one XMM-Newton pointing operated with the EPIC PN camera in small window mode (the same observation was already analysed by Bonnet-Bidaud et al. 2001). Their analysis mostly concentrated on the spectral evolution during dips, showing that current models (Church et al. 1998; Bonnet-Bidaud et al. 2001 models) are not able to account for the spectral changes during dips. We found similar difficulties in modeling the source emission even during "dip-free" intervals. This suggests that other spectral components are probably present especially at soft energies, and leads us to search for a new and different spectral model to describe the source emission. In the XMM-Newton observations analysed by Homan et al. (2003) clear "flaring" activity is present as demonstrated by the very high dynamical range in the $0.3-2 \mathrm{keV}$ lightcurves. The complexity of our BeppoSAX "dip-free" spectrum at soft energies is apparently unrelated to possible spectral mixing with flaring activity. Indeed, comparing the source variability seen with BeppoSAX (a factor of less than 2 compared to a factor $\sim 4$ in the XMM-Newton data) suggests that any flaring activity during BeppoSAX observation was less pronounced.

Acknowledgements. We would like to thank the anonymous referee for valuable comments and suggestions.

\section{References}

Boella, G., Chiappetti, L., Conti, G., et al. 1997, A\&AS, 122, 327

Bonnet-Bidaud, J. M., Haberl, F., Ferrando, P., et al. 2001, A\&A, 365, L282

Church, M. J., Bałucińska-Church, M., Dotani, T., \& Asai, K. 1998, ApJ, 504, 516

Cottam, J., Kahn, S. M., Brinkman, A. C., et al. 2001, A\&A, 365, $\mathrm{L} 277$

Cottam, J., Paerels, F., \& Mendez, M. 2002, Nature, 420, 51

Dickey, J. M., \& Lockman, F. J. 1990, ARA\&A, 28, 215

Done, C., Mulchaey, J. S., Mushotzky, R. F., \& Arnaud, K. A. 1992, ApJ, 395, 275

Frontera, F., Costa, E., Dal Fiume, D., et al. 1997, A\&AS, 122, 371

Gottwald, M., Haberl, F., Parmar, A. N., et al. 1986, ApJ, 308, 213

Homan, J., \& van der Klis, M. 2000, ApJ, 539, 847

Homan, J., Jonker, P., van der Klis, M., \& van Paradijs, J. 1999, ApJ, 516, L91

Homan, J., Wijnards, R., \& van den Berg, M. 2003, A\&A, 412, 799

Jimenez-Garate, M. A., Schulz, N. S., \& Marshall, H. L. 2003, ApJ, 590,432

Manzo, G., Guarrusso, S., Santangelo, A., et al. 1997, A\&AS, 122, 341

Mitsuda, K., Inoue, H., Koyama, K., et al. 1984, PASJ, 36, 741

Parmar, A. N., White, N. E., Giommi, P., \& Gottwald, M. 1986, ApJ, 308, 199

Parmar, A. N., Martin, D. D. E., Bavdaz, M., et al. 1997, A\&AS, 122, 309

Schulz, N. 1999, ApJ, 511, 304

Sunyaev, R. A., Churazov, E., Gilfanov, M., et al. 1991, A\&A, 247, L29

Sunyaev, R. A., \& Titarchuk, L. G. 1980, A\&A, 86, 121

Thomas, B., Corbet, R., Smale, A. P., et al. 1997, ApJ, 480, L21 